\title{
COMPARATIVO DA VIABILIDADE DA PROPOSTA DE UMA INCUBADORA
}

\section{COMPARISON OF THE VIABILITY OF AN INCUBATOR'S PROPOSAL}

Recebido em 20.09.2019 Aprovado em 10.12.2019

Avaliado pelo sistema double blind review

DOI: https://doi.org/10.32888/cge.v7i2.36590

\section{Angélica Baumann Cardoso}

baumann.angelica@gmail.com

Engenharia de Produção / UNIPAMPA - Universidade Federal do Pampa - Bagé/RS, Brasil

Caio Marcello Recart da Silveira

caiosilveiraa@,unipampa.edu.br

Engenharia de Produção / UNIPAMPA - Universidade Federal do Pampa - Bagé/RS, Brasil

Cláudio Sonáglio Albano

claudioalbano@,unipampa.edu.br

Administração Empresas / UNIPAMPA - Universidade Federal do Pampa - Bagé/RS, Brasil

\section{Resumo}

As micro e pequenas desempenham um importante papel no cenário econômico brasileiro. São reconhecidas as dificuldades que estas organizações encontram em seus primeiros momentos de existência, paralelo a este cenário uma incubadora de empresas é um ambiente flexível e encorajador, onde podem ser oferecidas algumas oportunidades para estes novos empreendimentos. Assim, este trabalho teve como objetivo verificar as condições regimentais e contextuais da incubadora de empresas proposta para o Campus Bagé (UNIPAMPA) e compará-las à estrutura e aos resultados de incubadoras instaladas em outros contextos. Com dados oriundos de fonte bibliográfica e documental, por intermédio de um estudo comparativo, os resultados revelam que alguns pressupostos permitem um desenvolvimento positivo, como: presença da incubadora dentro de uma Universidade; foco no aproveitamento dos recursos (laboratórios da Universidade) e foco no aproveitamento da formação de mão-de-obra realizada pela Universidade. Estes fatores convergem para a maximização da relação entre a incubadora e o contexto da Universidade.

Palavras-chave: Desenvolvimento Econômico. Incubadora. Universidade Federal.

\begin{abstract}
Micro and small enterprises play an important role in the Brazilian economic scenario. The difficulties that these organizations encounter in their first moments of existence are recognized, parallel to this scenario, a business incubator is a flexible and encouraging environment, where some opportunities can be offered for these new ventures. The objective of this study was to verify the organizational and contextual conditions of the business incubator proposed for Campé Bagé (UNIPAMPA) and to compare them to the structure and results of incubators installed in other contexts. With data from a bibliographic and documentary source, through a comparative study, the results reveal that some assumptions allow for a positive development, such as: presence of the incubator inside a University; focus on the use of resources (University laboratories) and focus on the use of the training of labor performed by the University. These factors converge to maximize the relationship between the incubator and the University context.
\end{abstract}

Keywords: Economic Development. Federal University. Incubator. 


\section{Introdução}

De acordo com SEBRAE (2016) as micro e pequenas empresas (MPEs) são responsáveis pela maioria dos empregos com carteira assinada do Brasil. Se somarmos a isso a ocupação que os empreendedores geram para si mesmos, pode-se dizer que os empreendimentos de micro e pequeno porte são responsáveis pela maioria dos empregos existentes no setor privado da economia. Dessa forma, a sobrevivência desses empreendimentos é condição importante para o desenvolvimento econômico do País. No entanto, esse mesmo estudo revela que aproximadamente $30 \%$ das micro e pequenas empresas brasileiras fecham as portas em até dois anos de atividade, e, a grande maioria não sobrevive por mais de cinco anos.

A cidade de Bagé, contexto deste trabalho, está situada na região sul (ou campanha) do estado do Rio Grande do Sul (RS). Segundo Chelotti (2006), a campanha gaúcha é um espaço regional inserido na região de fronteira brasileira com o Uruguai e Argentina, que sofreu ao longo das últimas décadas, principalmente a partir de meados da década de 80 , um profundo processo de perda de dinamismo econômico, resultado das dificuldades de inserção nos ciclos de expansão da economia brasileira. Há quase meio século, portanto, a região que já foi palco de empreendimentos pioneiros no Estado, defronta-se com dificuldades que têm colocado obstáculos ao seu desenvolvimento econômico e social.

A Universidade Federal do Pampa (UNIPAMPA) foi criada pelo governo federal, tendo como um de seus objetivos, o de colaborar para minimizar este processo de estagnação econômica que a região da campanha está sofrendo. Surgiu com a missão de ofertar ensino superior, desenvolver pesquisa nas diversas áreas do conhecimento e promover a extensão universitária, caracterizando sua inserção regional mediante atuação multicampi (em 10 cidades da região).

A presença de instituições de Ensino Superior em uma região é um elemento que contribui para seu desenvolvimento econômico e social, bem como de melhoria da qualidade de vida da população, uma vez que pode proporcionar o aproveitamento das potencialidades locais. Da mesma forma, os municípios que possuem representações de instituições de ensino superior (entre estas as universidades) podem desfrutar de um processo de transformação econômica, social e cultural. Este fato é alicerçado por parcerias firmadas entre essas instituições e as comunidades em que estão inseridas, fomentando a troca de informações e a interação científica, tecnológica e intelectual (UNIPAMPA, 2010).

Webster e Etzkowitz (1991), já relatavam que a ampliação da interação universidade-empresa estaria levando a universidade a incorporar as funções de desenvolvimento econômico às suas já clássicas atividades de ensino e pesquisa. Neste contexto, é que diversos mecanismos têm sido observados no âmbito desta relação como ferramenta de política industrial, tecnológica e de promoção do desenvolvimento local e regional, um destes mecanismos são as chamadas incubadoras de empresas. Conforme a Associação Nacional de Entidades Promotoras de Empreendimentos e Tecnologias Avançadas (ANPROTEC, 2002) uma incubadora de empresas é um ambiente flexível e encorajador, onde são oferecidas uma série de facilidades para novos empreendimentos. Os benefícios gerados atingem todos os envolvidos por instituições ensino superior, empreendedores, governos e respectivas comunidades.

A partir da situação de estagnação econômica da região da campanha e metade sul do estado do RS e possíveis benefícios que a incubadora de empresas pode gerar para a sociedade, o campus Bagé da UNIPAMPA deu início ao processo de implementação de uma incubadora. De acordo com o Artigo $2^{\circ}$ do regimento desta, o objetivo geral da Incubadora de Empresas do Campus Bagé é apoiar a formação e consolidação de micro e pequenas empresas de base tecnológica e/ou tradicionais que apresentem produtos, processos ou serviços inovadores, nos seus aspectos tecnológicos, gerenciais, mercadológicos e de recursos humanos, de modo a assegurar seu fortalecimento e a melhoria de seu desempenho, e em Parágrafo Único ressalta que a Incubadora de Empresas da UNIPAMPA Campus 
Bagé prioriza o desenvolvimento de novos negócios de acordo com as suas áreas de conhecimento (CBAGÉ, 2016).

Historicamente, empresas geradas em incubadoras têm maiores chances de sucesso que outras criadas fora deste ambiente. Este fato pretende reverter as dificuldades apontadas no início deste tópico pelas jovens empresas (geralmente micro e pequenas). Assim, a criação da Incubadora de Empresas na Unipampa Campus Bagé, deve ser mais um aliado na tentativa de mudar a realidade da região.

Assim, o objetivo deste trabalho é verificar as condições regimentais e contextuais da incubadora de empresas proposta para o Campus Bagé (UNIPAMPA) e compará-las à estrutura e aos resultados de incubadoras instaladas em outros contextos, caracterizando-se, portanto, como um estudo de caso que se utiliza do método comparativo de pesquisa, a partir de pesquisa bibliográfica e documental.

Nos próximos tópicos serão apresentados os pressupostos teóricos que sustentam o desenvolvimento do trabalho. Os procedimentos metodológicos utilizados para coleta e análise dos dados são descritos no tópico de número 5 , assim como no tópico 6 estão demonstrados os resultados e respectivas análises. Finalmente, no último tópico, são apresentadas as conclusões do trabalho, limitações e sugestões para trabalhos futuros.

\section{Incubadora de Empresas}

O sistema de incubação de empreendimentos iniciou-se em meados do século passado em alguns países da Europa e América do Norte. As primeiras políticas públicas de apoio aos parques tecnológicos e incubadoras de empresas, no Brasil foram desenvolvidas na década de 80 e apresentando um crescimento gradativo anualmente. As incubadoras podem ser classificadas em organizações públicas ou sem fins lucrativos, organizações acadêmicas, híbridas, privadas, etc (SILVA, 2009). Geralmente são formadas por intermédio de convênios ou termos de cooperação entre várias instituições comprometidas com o desenvolvimento de uma região como universidades, institutos de pesquisa, prefeituras, empresas privadas e associações de classe (ANPROTEC, 2013).

Outra definição de incubadora de empresas pode ser encontrada em ANPROTEC (2002), em que se apresenta como um ambiente flexível e encorajador, onde é oferecida uma série de facilidades para o surgimento e crescimento de novos empreendimentos. Além da assessoria na gestão técnica e empresarial da empresa, a incubadora oferece a infraestrutura e serviços compartilhados necessários para o desenvolvimento do novo negócio, como espaço físico, salas de reunião, equipamentos, entre outros. Reconhece ainda nas incubadoras funções catalisadoras do processo de desenvolvimento e consolidação de empreendimentos inovadores no mercado competitivo.

O objetivo de uma incubadora é de oferecer suporte a pequenas empresas (na fase inicial dos projetos) que buscam a diversificação e revitalização econômica, agregando valor aos seus produtos, viabilizando a interação com centros de ensino e pesquisa, para que a região beneficiada tenha maior produtividade e seja mais competitiva no mercado. Da mesma forma, propiciam o desenvolvimento de novos empreendimentos que sejam financeiramente viáveis e capazes de se adaptar ao mercado após o período de permanência na incubadora.

Além disso, apóiam a transformação de empresários potenciais em empresas crescentes e lucrativas e diminuem os riscos dos empreendimentos. As incubadoras também contribuem para a revitalização das regiões onde atuam, tendo em vista que favorecem o surgimento de novas empresas e empregos melhorando a distribuição de renda (RIBEIRO, 2004). Seus principais estimuladores acreditam que elas são uma opção inteligente para o desenvolvimento socioeconômico local e nacional. Proporcionam, portanto, duas motivações de naturezas distintas caracterizadas pela econômica e a social (SILVA, 2009).

O processo de incubação de empresas no Brasil é dividido em (i) incubadoras de base tecnológica, (ii) incubadoras tradicionais, (iii) incubadoras mistas, (iv) incubadoras sociais e (v) incubadoras de cooperativas. As incubadoras de base tecnológica compreendem as empresas cujos produtos, processos ou serviços nos quais a tecnologia representa alto valor e agregado, enquanto as incubadoras 
tradicionais abrigam as empresas de diferentes setores da economia, que queiram agregar valor aos seus produtos, processos ou serviços, mas que utilizem tecnologia largamente difundida. As incubadoras mistas são aquelas que abrigam tanto empreendimentos de base tecnológica como de setores tradicionais da economia e as incubadoras sociais e de cooperativas congregam os empreendimentos oriundos de projetos sociais e associativos (ANPROTEC, 2013).

De acordo com um estudo realizado em 2016 pela Anprotec, em parceria com o Sebrae, o Brasil tem 369 incubadoras em operação, que abrigam 2.310 empresas incubadas e 2.815 empresas graduadas, gerando 53.280 postos de trabalho. $\mathrm{O}$ faturamento das empresas apoiadas por incubadoras ultrapassa os R $\$ 15$ bilhões (ANPROTEC, 2016).

O público alvo das Incubadoras são estudantes, cientistas, empreendedores e empresas que desejam desenvolver novos projetos, produtos e serviços baseados em tecnologia inovadora. Geralmente cada usuário paga uma taxa para usufruir de todos esses serviços que é, normalmente, inferior ao preço que cada uma dessas despesas representaria para o empresário caso ele estivesse em outro ambiente. Quanto à carga tributária, a empresa incubada deve pagar todos os tributos como qualquer outra que não faça parte de uma incubadora (ANPROTEC, 2016).

Para fazer parte do processo de incubação é necessário cumprir alguns requisitos, e esse é um dos primeiros desafios enfrentados pelos empreendedores que buscam na incubadora o suporte necessário para o crescimento do negócio. Dentre os critérios avaliados, que podem variar de acordo com a incubadora, encontram-se a viabilidade do negócio, características inovadoras do produto, tecnologia do produto, perfil do empreendedor, setor de atuação do empreendimento, geração de emprego, entre outros fatores (RAUPP, 2006).

Os empreendimentos selecionados para as incubadoras brasileiras podem encontrar-se em uma das seguintes situações: Incubação à distância; Pré-incubação; Empresa incubada; Empresa associada e Empresa graduada. Entende-se por pré-incubação (também conhecido como Hotel de Projetos, Hotel de Ideias e Hotel Tecnológico) um conjunto de atividades que busca estimular o empreendedorismo e preparar os projetos que tenham potencial de negócios em empresas, durante um curto período (de 6 meses a um ano no máximo), para ingresso na incubadora.

A ênfase é dada ao plano de negócios, à pesquisa de mercado e à preparação dos empreendedores sobre gestão de negócios. Depois de passar pelo processo de seleção, a empresa passa para a fase de incubação (ou empresa residente), na qual desenvolve produtos ou serviços inovadores e recebe o apoio técnico, gerencial e financeiro de uma rede de instituições constituída, primordialmente, para criar e acelerar o desenvolvimento de pequenos negócios. O período de permanência nas incubadoras é, em média, de 3 (três) anos.

A Anprotec (2016) define como empresa associada (ou não residente) aquela que utiliza a infraestrutura e os serviços oferecidos pela incubadora, sem ocupar espaço físico, mantendo vínculo formal. A empresa associada busca o desenvolvimento de produtos e processos e o aprimoramento de suas ações mercadológicas. Ela tanto pode ser uma empresa recém-criada, como uma já existente no mercado. A empresa graduada (ou empresa liberada) é aquela organização que passou pelo processo de incubação e que alcançou desenvolvimento suficiente para ser habilitada a sair da incubadora. O vínculo com a incubadora pode manter-se na condição de empresa associada (SILVA, 2009).

\section{Incubadoras e Universidades}

As universidades possuem um papel importante no processo de inovação, pois são as maiores responsáveis por estimular a criatividade, podendo apoiar processos que venham a desenvolver (ou aprimorar) tecnologias. $\mathrm{O}$ vínculo estabelecido entre as incubadoras e as instituições de ensino quase sempre se realiza por meio de programas de extensão. A extensão universitária ou acadêmica pressupõe uma ação junto à comunidade, disponibilizando ao público externo da Instituição o conhecimento adquirido com o ensino e à pesquisa desenvolvidos pelas universidades e demais instituições de ensino. As incubadoras são vistas como programas de extensão diferente daqueles já 
praticados pelas instituições de ensino e pesquisa brasileiras, (SILVA, 2009).

Os benefícios gerados pelas incubadoras de empresas podem ser amplos para todos os envolvidos. Nas universidades, os principais benefícios são a conquista de novos alunos e reconhecimento como uma universidade tecnológica, empreendedora e inovadora, e a possibilidade de alunos realizarem estágios (e outras atividades) dentro das próprias incubadoras, aplicando os conhecimentos obtidos nos currículos dos cursos de graduação e/ou pós-graduação.

Para o governo, percebe-se a redução na mortalidade das empresas e a geração de retorno financeiro sob a forma de impostos; promoção da diversificação da economia local-regional, atuando como uma plataforma de desenvolvimento ao buscar potencializar as vocações regionais e suprimir a deficiência das cadeias produtivas locais; geração de emprego e renda; e o fortalecimento do espírito associativista. Para os empreendedores, gera a redução do custo e riscos do processo de inovação; estabelecimento de redes e alianças estratégicas; criação de uma cultura de gestão, inovação e planejamento; redução de custos operacionais e de parte das atividades gerenciais e o aumento gradativo do conhecimento teórico-prático. Para a comunidade local as vantagens envolvem o incremento de novos postos de trabalho e geração de renda; impulso e revitalização da economia local e a implementação de novas redes de negócios (SILVA, 2009).

Maculan e Merino (1998) realizaram um estudo sobre a avaliação da transferência do conhecimento na interação universidade-empresa, no qual analisaram duas experiências ocorridas entre grupos de pesquisa do Instituto Alberto Luiz Coimbra de Pós-Graduação e Pesquisa de Engenharia, da Universidade Federal do Rio de Janeiro (COPPE/UFRJ) e o Centro de Pesquisa Leopoldo Miguez de Mello (CENPES). Nesse estudo, os autores apresentam essa interação não como uma mera troca de produtos e serviços, mas sim como uma relação cujo principal objetivo é o de incrementar a base de conhecimentos para ambas as instituições.

Estudos mais recentes confirmam os benefícios e vantagens que podem resultar da relação entre Incubadoras e Universidades. Martins et. al, (2006) em um estudo sobre a relação entre Incubadoras de base tecnológicas e Universidades do estado de Minas Gerais (MG/Brasil), afirma que a localização das incubadoras próximas às universidades facilitam a interação entre o setor acadêmico e o setor produtivo.

Azevedo et. al, (2016), em estudo semelhante mas em todo o território brasileiro, além de reafirmarem o citado no estudo do parágrafo anterior relatam que na área de infraestrutura é fundamental o apoio das universidades, que permitem o acesso a salas de reuniões, auditórios, laboratórios, bibliotecas, redes de internet, entre outros recursos.

Na perspectiva da empresa, esse incremento do conhecimento deve viabilizar a solução de problemas nas práticas operacionais e o desenvolvimento de novos produtos ou processos com vistas a sua comercialização. Para a universidade, a interação deve viabilizar a diversificação das formas de valorização dos conhecimentos e competências acumuladas, a aquisição de novas competências, além de permitir que ela assuma um novo papel no crescimento econômico. E é justamente esse tipo de interação que a incubadora de empresas oferece.

\section{Contexto do trabalho: A Unipampa campus Bagé}

A UNIPAMPA faz parte do programa de expansão das universidades federais no Brasil, e foi criada pelo governo federal com o propósito de minimizar o processo de estagnação econômica da metade sul do Rio Grande do Sul e, desta forma, ser um agente da definitiva incorporação da região ao mapa do desenvolvimento do estado. Possui unidades acadêmicas em 10 municípios da metade sul do Rio Grande do Sul, com uma população de aproximadamente 2,6 milhões de pessoas, distribuídas por mais de 100 municípios.

Conforme consta em seu Plano de Desenvolvimento Institucional (PDI, 2014) a UNIPAMPA tem como missão a integração entre ensino, pesquisa e extensão, e assume a missão de promover a educação superior de qualidade, com vistas à formação de sujeitos comprometidos e capacitados a 
atuarem em prol do desenvolvimento regional, nacional e internacional. Ainda segundo este documento sua visão corresponde a constituir-se como instituição acadêmica de reconhecida excelência, integrada e comprometida com o desenvolvimento e, principalmente, com a formação de agentes para atuar em prol da região, do país e do mundo.

Assim fica claro, que uma das metas da UNIPAMPA é contribuir para mitigar o processo de estagnação econômica que se instalou nesta região na metade do século passado, em parte, por fatores climáticos que fizeram com que a principal atividade econômica, a agropecuária, ficasse sazonal, com períodos de perdas. Tal realidade implicou em prejuízos para vários segmentos das cidades que compõem esta localidade. A falta de indústrias que absorvessem a produção e oportunizassem a oferta de empregos também contribuiu para o empobrecimento da região.

No Campus de Bagé, atualmente, estão em funcionamento 11 cursos de graduação, sendo 5 da área de Engenharias (Alimentos, Computação, Energia, Produção e Química) e 6 na áreas das Licenciaturas (Física, Letras/Português, Letras/Inglês e Espanhol, Matemática, Música e Química). Também encontram-se 5 cursos de mestrado (em ambas as áreas - Engenharias e Licenciaturas), além de alguns cursos de especialização. Neste Campus existem diversos laboratórios, que podem contribuir com as atividades de uma incubadora de empresas, assim como dezenas de projetos de pesquisa, ensino e extensão (Gestão, 2018).

Após uma década de presença na região, a Universidade começa a promover uma mudança cultural, social e econômica na região. Como exemplo pode-se citar que no Campus Bagé todos os cursos de Engenharias já possuem respectivas empresas juniores estabelecidas e atuantes.

Outros dois fatos que podem ser citados como contribuições da Universidade são a qualidade dos egressos na área de Licenciaturas (cursos de graduação e pós-graduação), que contribuem para melhor qualidade da educação; e os egressos na área das Engenharias (também na graduação e pós-graduação) começam a mudar o perfil da qualidade da mão-de-obra disponível na região, além do potencial para contribuir com o surgimento de novos negócios. Os fatos citados acima certamente, deverão formar um circulo, ainda mais, virtuoso se aliados a um processo de incubadora de empresas.

\section{Procedimentos metodológicos}

A pesquisa caracteriza-se como um estudo de caso comparativo, busca analisar no regimento da incubadora informações como estrutura, resultados, benefícios e outras características e que será implementada no Campus Bagé da UNIPAMPA e compará-las às informações disponíveis a respeito de incubadoras já estabelecidas em contextos semelhantes.

Bruyne, Herman e Schoutheete (1977) afirmam que o estudo de caso justifica sua importância por reunir informações numerosas e detalhadas que possibilitem apreender a totalidade de uma situação. A riqueza das informações detalhadas auxilia o pesquisador num maior conhecimento e numa possível resolução de problemas relacionados ao assunto estudado.

Gil (2009) ressalta que o conceito de caso ampliou-se, a ponto de poder ser entendido como um grupo social, uma organização, um conjunto de relações, um papel social, um processo social, uma comunidade, uma nação ou mesmo toda uma cultura. Segundo FACHIN (2001) o método comparativo consiste em investigar coisas ou fatos e explicá-los segundo suas semelhanças e suas diferenças. Permite a análise de dados concretos e a dedução de semelhanças e divergências de elementos constantes, abstratos e gerais, propiciando investigações de caráter indireto.

A coleta dos dados incluiu a pesquisa bibliográfica e a pesquisa documental. A primeira pode ser definida como desenvolvida com base em materiais já elaborados e está representada pelo uso de artigos científicos, especificamente estudos de caso. A segunda, que difere da pesquisa bibliográfica pela natureza das fontes - definida pelo uso de documentos, está presente pela análise de toda documentação referente a incubadora de empresas do Campus Bagé.

Para a seleção dos casos foi realizada uma pesquisa em periódicos da área de Administração durante $\mathrm{o}$ ano de 2016. Nesta pesquisa foram selecionados trabalhos que abordassem incubadoras de empresas e que resultou em 21 trabalhos selecionados. 
Após esta fase, estes trabalhos passaram por um segundo processo de seleção onde foram selecionados casos de incubadoras que possuíssem contextos semelhantes ao da incubadora do Campus Bagé. Os critérios de comparação foram (I) contexto local; (II) objetivo da incubadora; (III) localização, infraestrutura e provisão de serviços; (IV) seleção de empresas; (V) equipe da incubadora; (VI) capitalização; (VII) fatores de alavancagem; e, (VIII) desenvolvimento local. Os trabalhos selecionados, bem como o critério utilizado, encontram-se descritos no Quadro 01.

Quadro 01 - Incubadoras selecionadas e critérios utilizados para escolha.

\begin{tabular}{|l|l|}
\hline Incubadora & Critério \\
\hline $\begin{array}{l}\text { Incubadora de Empresas de Santa Rita do } \\
\text { Sapucaí - MG }\end{array}$ & $\begin{array}{l}\text { Características semelhantes entre os dois municípios (Bagé e Santa } \\
\text { Rita do Sapucai) }\end{array}$ \\
\hline Incubadora CENTEV/UFV & Estar inserida em uma Universidade Federal \\
\hline Incubadora Tecnológica de Caxias do Sul & Estar situada no estado do Rio Grande do Sul \\
\hline Incubadora de Empresas do CEFET/RJ & Estar inserida em uma Universidade Federal \\
\hline $\begin{array}{l}\text { Incubadora de Empresas de Base Tecnológica da } \\
\text { UNISINOS }\end{array}$ & $\begin{array}{l}\text { Estar situada no estado do Rio Grande do Sul e em uma } \\
\text { Universidade. }\end{array}$ \\
\hline
\end{tabular}

Fonte: autores do trabalho

Para análise dos dados, foram utilizados os pressupostos da análise de conteúdo. Para Bardin (2002), esta compreende um procedimento sistemático e objetivo de descrição do conteúdo, uma das formas de operacionalizar a análise de conteúdo é realizar a categorização dos textos advindos da coleta de dados. Essa categorização pode ser realizada a priori, conforme a fundamentação teórica.

Assim, as categorias devem ser válidas, uma categorização válida deve ser significativa e coerente com os objetivos do trabalho. Adotou-se uma postura de tratamento qualitativo para análise dos dados, na qual se buscou identificar a ocorrência de citações ou referências às categorias previamente estabelecidas (CRESWELL, 2009).

Neste trabalho a classificação das categorias atendeu os critérios de comparação (citados anteriormente) e dentro destes por palavras ou expressões que permitissem a comparação entre as realidades estudadas.

\section{Apresentação e análise dos resultados}

Os resultados e respectivas análises estão dispostos na ordem que os critérios de comparação foram expostos no tópico procedimentos metodológicos. Optou-se por esta forma de expor os resultados, por entender que esta facilita a leitura e entendimento. Importante destacar que para cada critério comparado, foi selecionado um (ou mais) trabalho para a comparação. Um mesmo trabalho pode ter sido utilizado para comparações em um ou mais critérios.

Assim, com relação ao critério contexto local, foi selecionado o caso da incubadora do município de Santa Rita de Sapucaí (MG). Antes da instalação da incubadora, sua economia era predominantemente agropecuária, apresentava graves problemas em relação ao desemprego e êxodo de jovens (mão-deobra) para outras regiões, Todas estas características são semelhantes a cidade (e região) de Bagé.

Por intermédio de parceria com o poder público a instalação da incubadora gerou novos postos de trabalho, crescimento da renda, melhora na qualidade de vida, entre outros benefícios. Da mesma forma, estas parcerias estão previstas na proposta para a incubadora do Campus Bagé, como o objetivo de estimular o empreendedorismo, utilizando diversos recursos necessários (disponíveis na Universidade, como seus laboratórios) para o desenvolvimento dos novos negócios, além da formação 
de mão-de-obra com qualidade em suas mais diversas atividades de ensino, pesquisa e extensão.

Com relação ao objetivo da incubadora, foram selecionados dois casos: a incubadora de Santa Rita do Sapucaí (MG) e a incubadora CENTEV/UFV. Os objetivos tanto das incubadoras citadas quanto da incubadora de Bagé são bastante parecidos: fortalecer o desenvolvimento local, amenizar o desemprego, aumentar qualidade de vida e criar e fortalecer empresas de base tecnológica. Esta grande aproximação entre os objetivos, pode ser explicada pela forte semelhança de contextos entre os dois casos escolhidos e o contexto da incubadora do Campus Bagé.

Para o critério localização, infraestrutura e provisão de serviços, foram selecionados para comparação os quatro casos de incubadoras localizadas em Universidades, exatamente por este fator. Este fato, localização dentro de uma Universidade, segundo os casos, facilita a contribuição da universidade no sentido de inovações tecnológicas, beneficiando as empresas incubadas.

Mais especificamente, com relação à infraestrutura e serviços prestados, todas procuram oferecer um espaço físico com ambiente compartilhado configurado para atender as necessidades das empresas incubadas. Em relação aos serviços, prestam consultorias, seminários, treinamentos, palestras e orientação empresarial, de maneira geral, com algumas pequenas variações de uma incubadora para outra.

A convergência desta possibilidade de interação entre Universidade e Incubadora, demonstra-se como um fator positivo relevante, visto que empresas incubadas podem fazer uso das estruturas e conhecimento disponíveis nas Universidades.

Para análise do critério seleção de empresas foi selecionado o caso da CEFET/RJ. Esta incubadora e a proposta da incubadora do Campus Bagé, apresentam um processo seletivo semelhante. Existe a divulgação de edital para selecionar propostas, e as empresas serão selecionadas se estiverem de acordo com os critérios definidos no edital e enquadradas nas áreas definidas pelo Conselho Diretor da incubadora. Além dos critérios estabelecidos, as equipes deverão atender às exigências expressas no Contrato de Adesão do Sistema de Incubação.

Também o caso do CEFET/RJ, foi selecionado para análise do critério equipe da incubadora. Neste quesito existem algumas diferenças, enquanto na proposta da incubadora do Campus Bagé está previsto um conselho diretor, no caso do CEFET/RJ, existe um único gerente. No entanto, a incubadora do CEFET/RJ conta em sua equipe com o diferencial de assessores em áreas especializadas, o que a princípio não está especificado no regimento da incubadora bageense.

Convém destacar que a incubadora bageense conta, em seu conselho diretor, com docentes, e os mesmos podem ser especialistas em diferentes áreas. Este critério é importante no suporte que esta equipe fornece às empresas incubadas, não há como comparar resultados deste diferencial, pois a incubadora de Bagé ainda não está em funcionamento, mas considerando as áreas de conhecimento dos docentes, e que a incubadora de Bagé busca beneficiar negócios desenvolvidos dentro das áreas trabalhadas nos cursos de graduação da UNIPAMPA, pode-se prever que os resultados atingidos pela equipe possam ser satisfatórios.

A incubadora de CENTEV/UFV foi escolhida para análise do critério capitalização. Ambas utilizam (no caso do Campus Bagé - deve utilizar), um sistema de capitalização no qual os responsáveis pelos projetos pré-incubados e pelas empresas participantes dos programas pagam à incubadora, mediante apresentação de Guia de Recolhimento da União (GRU), pelo uso das instalações físicas dessa e seus serviços complementares.

Com relação ao critério fatores de alavancagem, a proposta da incubadora do Campus Bagé, foi comparada com outras três: Santa Rita do Sapucaí (MG), Incubadora Tecnológica de Caxias do Sul e Incubadora Tecnológica da UNISINOS. O que estimulou a criação da incubadora de Bagé é proporcionar um ambiente adequado para os alunos da própria universidade desenvolverem novos negócios, diminuindo o êxodo de profissionais habilitados para outras regiões. Apesar de cada incubadora surgir de uma problemática diferente, todas compartilham basicamente de um objetivo, semelhante a proposta da incubadora de Bagé.

A UNISINOS criou à incubadora pois buscava ampliar a sua presença e participação no atendimento às diferentes necessidades regionais e desenvolvimento das potencialidades locais, consolidando o 
papel da universidade como agente do desenvolvimento local. O motivo que levou à criação da Incubadora pela Universidade de Caxias do Sul foi a necessidade de agregar os setores industriais e acadêmico, gerando visibilidade para a própria universidade como também tornando empresas capazes de competir no mercado. No caso de Santa Rita do Sapucaí, a incubadora foi planejada como uma solução para os problemas que o município vinha enfrentando, onde um dos principais era a elevada taxa de desemprego.

O trabalho que abordou de forma clara a questão do desenvolvimento local, foi o caso da incubadora CENTEV/UFV. Comparando este caso com a proposta do Campus Bagé, percebe-se que ambas deixam claro que desejam contribuir para geração de emprego e renda; formação de recursos humanos qualificados; formação de empreendedores; difusão de uma cultura empreendedora na região; criação de um mercado de trabalho dinâmico entre empresas de tecnologia avançada; e finalmente para atração de investidores.

\section{Considerações finais}

Este estudo teve como objetivo verificar as condições regimentais e contextuais da incubadora de empresas proposta para o Campus Bagé (UNIPAMPA) e compará-las, por intermédio de pesquisa documental e bibliográfica, à estrutura e os resultados de incubadoras instaladas em contextos com alguma semelhança.

A comparação foi realizada conforme critérios estabelecidos. Podem-se destacar como principais fatores que contribuíram para o atendimento dos objetivos das incubadoras, e presentes na proposta da incubadora do Campus Bagé, a presença da incubadora dentro de uma Universidade; foco no aproveitamento dos recursos materiais (por exemplo, laboratórios da Universidade) e foco no aproveitamento da formação de mão-de-obra realizada pela Universidade. Estes últimos fatores convergem para a maximização da relação entre a incubadora e o contexto da Universidade.

Foi possível perceber condições bastante próximas das incubadoras em funcionamento com relação à proposta da incubadora do Campus Bagé. Assim, é possível afirmar que estão postas as condições regimentares e estruturais para que a incubadora do Campus Bagé possa efetivamente atingir seus objetivos.

Pode-se citar como um limite do trabalho, o fato de que não foi possível realizar comparações sobre possíveis resultados negativos quando da implantação e desenvolvimento das incubadoras com relação a proposta do Campus Bagé, visto esta ser uma proposta e ainda não estar funcionando, quando da realização deste estudo. Assim, visando atender a esta limitação sugere-se como um trabalho futuro realizar esta comparação com estas mesmas incubadoras, mas fazendo coleta de dados com entrevistas ou estudos de casos.

As incubadoras contribuem para a revitalização das regiões onde atuam, tendo em vista que favorecem o surgimento de novas empresas e empregos melhorando a distribuição de renda. Diante da situação de estagnação econômica atual da região do pampa, e do objetivo da Universidade Federal do Pampa, que é justamente intervir neste quadro, a implementação de uma incubadora pode gerar muitos benefícios para todos os envolvidos e representados pelo governo, universidade e sociedade em geral.

\section{Referências}

ANPROTEC. ASSOCIAÇÃO NACIONAL DE ENTIDADES PROMOTORAS DE EMPREENDIMENTOS INOVADORES. SERVIÇO BRASILEIRO DE APOIO ÀS MICRO E PEQUENAS EMPRESAS. Planejamento e implantação de incubadoras de empresas. Brasília. 2002.

ASSOCIAÇÃO NACIONAL DE ENTIDADES PROMOTORAS DE EMPREENDIMENTOS INOVADORES. Ministério da Ciência, Tecnologia e Inovação. - Brasília. 2013. 
ANPROTEC. ASSOCIAÇÃO NACIONAL DE ENTIDADES PROMOTORAS DE EMPREENDIMENTOS INOVADORES. SERVIÇO BRASILEIRO DE APOIO ÀS MICRO E PEQUENAS EMPRESAS. Estudo de impacto econômico: Segmento de incubadoras de empresas do Brasil. 2016.

AZEVEDO, I. S. C., POZZOBON, C. CAMPOS, J. G. URRUTIA, S. L. TEIXEIRA, C. S. Análise das Incubadoras Universitárias do Brasil. 26 Conferência da ANPROTEC. Fortaleza/Ceará. Outubro 2016.

BARDIN, Laurence. Análise de Conteúdo. Tradução de Luís Antero Reto e Augusto Pinheiro. Lisboa: Edições 70, 2002.

BRUYNE, P., Herman, J., \& SCHOUTHEETE, M. (1977). Dinâmica da pesquisa em ciências sociais:os polos da prática metodológica. Rio de Janeiro:Francisco Alves.

CBAGÉ (2016). Regimento Incubadora de Empresas do Campus Bagé/UNIPAMPA. Disponível em: http://porteiras.r.unipampa.edu.br/portais/consuni/files/2016/05/Res.-160_2016-

Cria\%C3\%A7\%C3\%A3o-e-Regimento-Incubadora-de-Empresas-Campus-Bag\%C3\%A9.pdf.

Acesso em Maio de 2018.

CHELOTTI, Marcelo C. Revisitando a questão regional na Campanha Gaúcha: a incorporação de novos elementos no pós 1990. Caminhos da Geografia. Instituto de Geografia UFU, MG, Disponível em: $\quad<$ set/2018, http://www.seer.ufu.br/index.php/caminhosdegeografia/article/viewFile/15399/8698>

CRESWELL, J. W. Projeto de pesquisa. Método qualitativo, quantitativo e misto. $3^{\text {a }}$ edição. Porto Alegre: editora Artmed, 2009.

GIL, A. C. Como elaborar projetos de pesquisa. 4. ed. São Paulo: Atlas, 2009. 175 p.

GESTÃO, 2017. Relatório de Gestão Campus Bagé 2017. Disponível em: http://porteiras.r.unipampa.edu.br/portais/proplan/files/2018/07/relatorio_gestao_2017-bage.pdf. Acesso em Agosto de 2018.

FACHIN, O. Fundamentos de metodologia. 3. ed. São Paulo: Atlas, 2001.

MACULAN, Anne-Marie; MERINO, José Carlos Alvarez. Como avaliar a transferência do conhecimento na interação universidade - empresa? In: XX SIMPÓSIO DE GESTÃO DA INOVAÇÃO TECNOLÓGICA, 1998, São Paulo. Anais... São Paulo: 1998. p. 802-812.

MARTINS, G. S. XAVIER, W. S. LIMA, A. A. T. F. C. OLIVEIRA, R. GAVA, R. A Interação Universidade/Empresa nas Incubadoras de Empresas de Base Tecnológica de Minas Gerais. XXIV Simpósio de Gestão da Inovação Tecnológica. ANPAD. 2006. Gramado. Rio Grande do Sul.

SERVIÇO BRASILEIRO DE APOIO ÀS MICRO E PEQUENAS EMPRESAS. Participação das micro e pequenas empresas na economia brasileira. Sebrae, Brasília/DF, p 17, 2014.

ROMERO, Cristiano, O Primeiro Passo. Jornal do Brasil, Rio de Janeiro, 25 de novembro de 1999.

PDI (2014). Plano Desenvolvimento Institucional da UNIPAMPA, 2014-2018. Disponível em: http://porteiras.r.unipampa.edu.br/portais/consuni/files/2010/06/Res.-71_2014-PDI.pdf. Acesso em Agosto de 2018.

RAUPP, F. M.; BEUREN, I. M. O suporte das incubadoras brasileiras para potencializar as características empreendedoras nas empresas incubadas. Revista de Administração, v. 41, n. 4, p. 419430, 2006. Disponivel em: $<$ set/2018, http://www.revistas.usp.br/rausp/article/view/44416/48036>.

RIBEIRO, Simone Abreu; ANDRADE, Raphael Medina Gomes de; ZAMBALDE, André Luiz. 
Incubadoras de empresas, inovação tecnológica e ação governamental: o caso de Santa Rita do Sapucaí (MG). Cad. EBAPE.BR [online]. 2005, vol.3, n.spe, pp.01-14.

SILVA, S. A. A relevância das incubadoras de empresas no mundo contemporâneo. Ponto e vírgula, 6: 235- 251, 2009.

SEBRAE. SERVIÇO BRASILEIRO DE APOIO ÀS MICRO E PEQUENAS EMPRESAS. Sobrevivência das empresas no Brasil. Brasília, DF, p 4-5, Outubro 2016.

UNIVERSIDADE FEDERAL DO PAMPA. Projeto Pedagógico Curso de Engenharia de Produção 2010. Universidade Federal do Pampa Disponivel em: http://www.unipampa.edu.br/portal/documentos/doc_details/338. Acesso em junho de 2018.

WEBSTER, A.J. e ETZKOWITZ, H. Academic-industry relations: the second academic revolution? Londres, Science Policy Support Group, 1991, 31p. 